Artigo original

Hegemonia - Revista Eletrônica de Relações Internacionais do Centro Universitário Unieuro

ISSN: 1809-1261

UNIEURO, Brasília, número 7, 2011, pp. 5-38.

Recebido em: 1/11/2010

Revisado em: $3 / 12 / 2010$

Aprovado em: 22/12/2010

\title{
Será o fim das negociações comerciais agrícolas da Rodada de Doha?: Um estudo da última revisão do Projeto sobre Modalidades para a Agricultura (dezembro de 2008)
}

¿Hacia el fin de las negociaciones comerciales agrícolas en la Ronda de Doha?: Un análisis de la última revisión del Proyecto sobre Modalidades para la Agricultura (diciembre de 2008).

Brunetto, María J osé

Resumo: O objetivo deste artigo é o de analisar a última revisão do projeto sob as Modalidades para Agricultura, apresentada pelo presidente do Comitê da Agricultura como o espaço da negociação comercial, em sua reunião extraordinária de julho de 2007. Antes de começar com esta análise, é necessário fazer uma explicação precedente pela qual como no exemplo das revisões precedentes e de ambos os projetos já apresentados previamente à $V$ Conferença Ministerial de Cancun e em julho de 2006, esta nova revisão refleta as últimas reflexões nos assuntos da negociação agricola que surgem da acumulação progressiva das posições dos membros, das consultas informais feitas nos meses precedentes e das reuniões formais do Comitê da Agricultura no mês de setembro de 2008. Mais, ao contrário das revisões precedentes, o objetivo desta nova revisão é apresentar aos negociadores o conteudo possível do acordo final, pondo ênfase nos aspetos em que já existe convergência. Além disso e simultaneamente naqueles outros aspetos onde existem discrepâncias entre os membros, este original foi acompanhado por outros três documentos onde se fazevam sugestões sob como aproximar as posiçoes. Além do mais, uma outra pergunta

$1 \square$ Professora do Bacharelado em Relações Internacionais da Universidade da República (Uruguai). 
Artigo original

Hegemonia - Revista Eletrônica de Relações Internacionais do Centro Universitário Unieuro

ISSN: 1809-1261

UNIEURO, Brasília, número 7, 2011, pp. 5-38.

metodôlogica precedente, é que o trabalho acompanha a estrutura mesma do projeto. Esta é a razão pela qual o artigo consiste em três porções. Primeiramente, se faz a referência ao avanço das negociações na ârea do acesso aos mercados. Em segundo lugar, se apresentam os avanços feitos no pilar das reducçôes às ajudas internas impostas pelos paises para apoiar o desenvolvimento dos sectores agrícolas. No último lugar, se analisa a proposta das modalidades no âmbito da competência das exportações.

Palavras-chave: Rodada Doha; agricultura; negociçoes comerciais.

Resumen: El objetivo de este trabajo es analizar la última revisión del proyecto sobre las Modalidades, presentado por el Presidente del Comité de Agricultura como ambito de negociación comercial, en su reunión extraordinaria de julio de 2007. Sin embargo, antes de comenzar con este análisis, hay que realizar una aclaración previa que como en el caso de las revisiones anteriores y de los dos proyectos anteriores ${ }^{2}$, esta nueva revisión refleja las últimas reflexiones sobre los temas de la negociación agrícola que surgen de la acumulación progresiva de las posiciones de los miembros, de las consultas informales realizadas en los meses anteriores y las reuniones formales del Comité de Agricultura realizadas desde setiembre de 2008. Sin embargo, a diferencia de las revisiones anteriores, el objetivo de esta nueva revisión es presentar a los negociadores el posible contenido del conjunto final de resultados, poniendo énfasis los aspectos sobre los que ya existe convergencia. Además, simultáneamente sobre aquellos otros aspectos sobre los se mantenían las discrepancias entre los miembros, este documento se acompañaba de otros tres documentos en los que se hacía sugerencias sobre la forma de cómo abordar esos temas. Además, otra cuestión metodológica previa, es que el trabajo va a estar estructurado siguiendo la estructura misma del proyecto, razón por la cual el mismo consta de tres partes. La primera hace referencia al avance de las negociaciones en el pilar de acceso a los mercados. La segunda hace referencia al avance en el segundo pilar - programas de ayuda interna. La última analiza la propuesta de modalidades sobre competencia de exportaciones.

Palabras-Ilave: Ronda Doha; Agricultura; Negociaciones comerciales

$2{ }^{1}$ El primerio de los cuales fue presentada tempranamente en 2002 y el segundo en julio de 2006. 
Artigo original

Hegemonia - Revista Eletrônica de Relações Internacionais do Centro Universitário Unieuro

ISSN: 1809-1261

UNIEURO, Brasília, número 7, 2011, pp. 5-38.

\section{I ntroducción}

El proceso de reforma del Sistema Multilateral del Comercio en el sector agrícola se inició más tempranamente a la fecha de lanzamiento de la denominada Ronda de Doha para el Desarrollo en noviembre de 2001. Desde su inicio ha atravesado diferentes instancias y etapas, con muchos vaivenes, idas y vueltas. Si bien la ronda de negociaciones debía concluir en el año 2005, el período de negociaciones ha sido prórrogado en la VI Conferencia Ministerial de Hong Kong, debiendo concluir en 2007, siendo reiteradamente extendido.

Desde su comenzo, estas negociaciones se han dado en tres pilares de acuerdo al mandato otorgado en el Programa de Trabajo lanzado en la IV Conferencia Ministerial de Doha - mejorar el acceso a los mercados, reducciones sustanciales de las ayudas internas a la agricultura, y reducción sustancial para su posterior eliminación de las subvenciones a las exportaciones y establecimiento reglas claras para las políticas de competencia de las exportaciones.

A lo largo de este largo período, en tres momentos, las negociaciones estuvieron al borde del fracaso ( $V$ Conferencia Ministerial de Cancun de 2003, en la reunión del Consejo Central de Postdam de julio 2006 y en la Mini-Ministerial de Ginebra de julio de 2008) y cuan el ave fénix han resurgido con nuevas fuerzas y brios. 
Artigo original

Hegemonia - Revista Eletrônica de Relações Internacionais do Centro Universitário Unieuro

ISSN: 1809-1261

UNIEURO, Brasília, número 7, 2011, pp. 5-38.

Desde su suspensión temporal en el año 2006, el Presidente del Comité de Agricultura de la Organización Mundial del Comercio como grupo negociador ha presentado ha presentado dos proyectos para las Modalidades: uno en julio de 2006 y otro en julio de 2007, siendo el último de estos proyectos sobre el que se está negociando estas Modalidades. Desde su presentación en julio de 2007, este proyecto ha sufrido cuatro revisiones (una todavía en el año 2007 y las otras tres en 2008.

El objetivo de este trabajo es analizar esta última revisión del proyecto sobre las Modalidades de julio de 2007. Sin embargo, antes de comenzar con este análisis, hay que realizar una aclaración previa que como en el caso de las revisiones anteriores y de los dos proyectos anteriores ${ }^{3}$, esta nueva revisión reflejaba las últimas reflexiones sobre los temas de la negociación agrícola que surgían de la acumulación progresiva de las posiciones de los miembros, de las consultas informales realizadas en los meses anteriores y las reuniones formales del Comité de Agricultura realizadas desde setiembre de 2008. Sin embargo, a diferencia de las revisiones anteriores, el objetivo de esta nueva revisión es presentar a los negociadores el posible contenido del conjunto final de resultados, poniendo énfasis los aspectos sobre los que ya existe convergencia. Además, simultáneamente sobre aquellos otros aspectos sobre los se mantenían las discrepancias entre los miembros, este documento se

3 El primerio de los cuales fue presentada tempranamente en 2002 y el segundo en julio de 2006. 
Artigo original

Hegemonia - Revista Eletrônica de Relações Internacionais do Centro Universitário Unieuro

ISSN: 1809-1261

UNIEURO, Brasília, número 7, 2011, pp. 5-38.

acompañaba de otros tres documentos en los que se hacía sugerencias sobre la forma de cómo abordar esos temas.

Además, otra cuestión metodológica previa, es que el trabajo va a estar estructurado siguiendo la estructura misma del proyecto, razón por la cual el mismo consta de tres partes. La primera hace referencia al avance de las negociaciones en el pilar de acceso a los mercados. La segunda hace referencia al avance en el segundo pilar programas de ayuda interna. La última analiza la propuesta de modalidades sobre competencia de exportaciones.

\section{El primer pilar: Acceso a los mercados}

¿Cuáles son las mejoras presentadas por este último documento en esta temática? Como en las versiones anteriores, los productos son clasificados en cuatro categorías o bandas según el nivel arancelario consolidado que sirve como punto de partida para las reducciones a implementar, según una fórmula estratificada que queda plasmada en el siguiente cuadro. Esta fórmula preveía lo ya consagrado en la Conferencia Ministerial de Hong Kong, los recortes mayores para las bandas superiores y los menores para los niveles arancelarios de la banda inferior. 
Artigo original

Hegemonia - Revista Eletrônica de Relações Internacionais do Centro Universitário Unieuro

ISSN: 1809-1261

UNIEURO, Brasília, número 7, 2011, pp. 5-38.

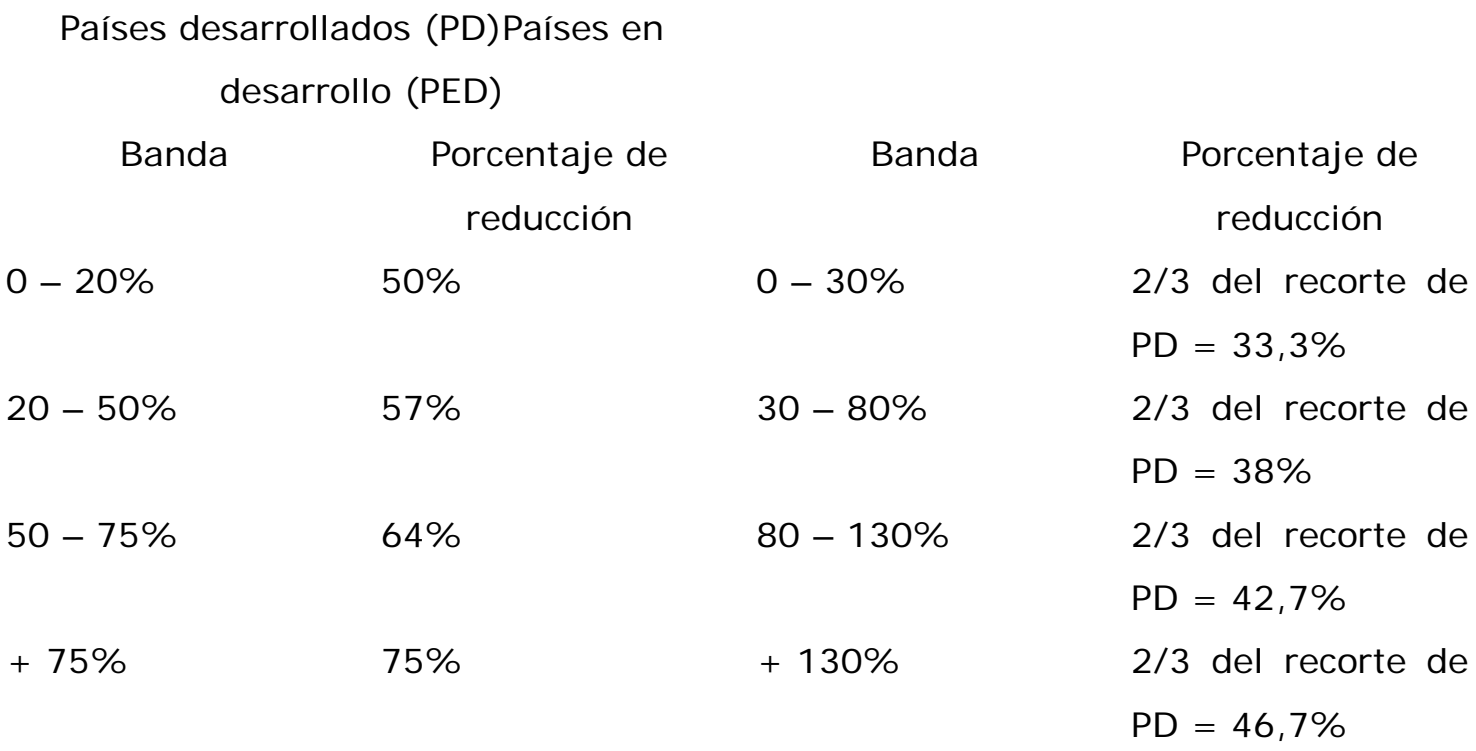

Reducción a realizar en 5 añosReducción a

realizar en 10 años

Reducción promedio: 54\%Reducción

promedio: $36 \%$

Reducciones a

realizarse en tramos

anuales iguales

Los porcentajes de recortes presentados en este documento eran bastante cercanos a los reclamados originalmente por el G-20. Además, cerca del $80 \%$ de las líneas los mayores recortes arancelarios tenían que ser hechos por la Unión Europea.

Además, como en los documentos anteriores, la reducción arancelaria no se aplicaría en la forma prevista a todos los productos, puesto que se preveía una cierta flexibilización de la regla general para ciertos productos, considerados políticamente "sensibles" y para aquellos productos que entraban dentro de la categoría de "productos 
Artigo original

Hegemonia - Revista Eletrônica de Relações Internacionais do Centro Universitário Unieuro

ISSN: 1809-1261

UNIEURO, Brasília, número 7, 2011, pp. 5-38.

especiales de los países en desarrollo", porque afectaban la seguridad alimentaria, la seguridad de los medios de subsistencia y el desarrollo rural de estos países y sobre todo de los países menos desarrollados.

Del mismo modo, dentro de los países en desarrollo, existía un tratamiento diferenciado más favorable en cuanto a porcentajes menores de reducción arancelaria y un recorte máximo promedio del $24 \%$, para aquellos países, cuyas economías eran consideradas pequeñas y vulnerables ${ }^{4}$ y para algunos países de reciente adhesión ${ }^{5}$ al sistema multilateral, o en cuanto a quedar exentos a la obligación de reducir aranceles para aquellos países considerados como países menos avanzados y para otros de los países de reciente adhesión, especialmente aquellos que se hubiesen incorporado al sistema hacía muy poco tiempo, o que fueran muy pequeños o de ingresos per cápita muy bajos.

Así como también para los países en desarrollo, la propuesta de reducción arancelaria incluida en el documento, les quitaba solo una parte del colchón protector dado por los aranceles, porque las reducciones arancelarias se aplicarían sobre los niveles consolidados y no sobre los efectivamente aplicados, que podían ser muy inferiores a los consolidados. De este modo, los recortes efectivos de los

4 Unos 45 países estaban expresamente enumerados en el Anexo I de este documento.

5 Para estos países, se preveía 10\% menos de reducción para los niveles arancelarios de las dos bandas superiores y $5 \%$ menos de recorte para los que se encontraban en las bandas inferiores. 
Artigo original

Hegemonia - Revista Eletrônica de Relações Internacionais do Centro Universitário Unieuro

ISSN: 1809-1261

UNIEURO, Brasília, número 7, 2011, pp. 5-38.

aranceles no se producirían, siendo inocuos sobre las condiciones de acceso a mercados de esos países.

En esta última revisión del Proyecto Falconer, sin fijar un tope arancelario, se mantenía el incentivo para que los países desarrollados ajustaran sus aranceles del estrato superior al 100\% como máximo y para que los países en desarrollo, lo hicieran al $150 \%$, previéndose un trato separado para los productos sensibles. Pues para estos productos, si bien se preveía la posibilidad de imponer aranceles superiores al $100 \%$ o al 150\% según el tipo de país, el porcentaje por encima del $100 \%$ o del $150 \%$ no podía ser superior al $1 \%$ y además, el mismo debía ser compensado con el otorgamiento de un contingente arancelario o la ampliación de uno ya existente en un $0,5 \%$ del consumo interno o con un recorte arancelario que se realizaría dos años antes de lo previsto o sería un $10 \%$ superior al normal.

Para atacar la progresividad arancelaria, que se daba cuando los aranceles aplicables a los productos con un grado mayor de elaboración son superiores a los aplicables a las materias primas o productos en bruto, este documento contemplaba que cuando el producto elaborado fuese objeto de este problema y dependiendo si el nivel arancelario consolidado se encontrase en los dos estratos inferiores, el recorte arancelario previsto debía ser 7\% superior al general y si dicho nivel se ubicara en los dos estratos superiores, el recorte arancelario aplicable debía ser un 6\% superior al general. Este mecanismo de corrección de la progresividad arancelaria, no se 
Artigo original

Hegemonia - Revista Eletrônica de Relações Internacionais do Centro Universitário Unieuro

ISSN: 1809-1261

UNIEURO, Brasília, número 7, 2011, pp. 5-38.

debía aplicar al producto elaborado si este producto era un producto sensible.

Como los documentos anteriores, este texto contemplaba dos opciones: la primera era que todos los aranceles se expresaran como aranceles ad-valorem simples y la segunda, que para un $10 \%$ de los productos se pudiese mantener aranceles de otro tipo, pero con ciertas condiciones. Si bien esto abría la posibilidad de no simplificar las estructuras arancelarias, por lo que para evitarlo se establecía que bajo ningún concepto, ninguna estructura arancelaria debía ser mas compleja que la vigente.

En virtud del Acuerdo de Agricultura se abría la posibilidad de que aquellos productos que antes de la vigencia del mencionado acuerdo, estuviesen sometidos a restricciones no arancelarias como las cuotas, pasasen a quedar sometidos a contingentes arancelarios, es decir restricciones cuantitativas en monto $o$ en volumen de las importaciones acompañadas por aranceles intra-cuota inferiores a los aranceles extra-cuota. Por lo que, la última revisión de diciembre de 2008 incorporaba disposiciones con respecto a los aranceles consolidados dentro de los contingentes, la magnitud de la reducción de estos aranceles y la posibilidad de creación de nuevos contingentes arancelarios con aranceles intra-cuota nulos. Con relación a los aranceles intra-cuota aplicados, se preveía su reducción que podía ser o de un $50 \%$ o al $10 \%$ (si el resultado fuese menor) en el caso de los países desarrollados, de un $15 \%$ en el caso de los países en desarrollo, del $10 \%$ en el de países de reciente adhesión y del $7,5 \%$ en el de economías pequeñas y vulnerables. Además se 
Artigo original

Hegemonia - Revista Eletrônica de Relações Internacionais do Centro Universitário Unieuro

ISSN: 1809-1261

UNIEURO, Brasília, número 7, 2011, pp. 5-38.

proponía que los aranceles de los países desarrollados que fuesen inferiores al $5 \%$ se eliminaran en el plazo de un año. Las disciplinas relativas a la administración de contingentes remitían a los procedimientos establecidos en materia trámites de licencias de importación.

Como se señalara una de las primeras formas de flexibilización de la aplicación de la regla general de reducción arancelaria es el tema de los productos sensibles. Como en documentos anteriores, la designación de productos sensibles quedaba disponible para todos los países. Para este conjunto de productos, se contemplaba unos porcentajes de reducción menores a los recortes generales establecidos por la fórmula para los demás productos. Como en el Paquete de Julio, se mantenía que hasta un nivel del $4 \%$ de las partidas arancelarias agrícolas de los países desarrollados y hasta uno del 5,3\% de las líneas arancelarias para los países en desarrollo, podía ser considerado como "producto sensible"".

Además, el texto ofrecía dos opciones para seleccionar a los productos sensibles: la primera era que los mismos ya estuvieran sometidas a una contingentación arancelaria en virtud de los compromisos nacionales asumidos al aprobar el Acuerdo sobre la Agricultura de la OMC y la segunda que fuesen nuevos productos. Proponía también las mismas magnitudes de desvío respecto al 
Artigo original

Hegemonia - Revista Eletrônica de Relações Internacionais do Centro Universitário Unieuro

ISSN: 1809-1261

UNIEURO, Brasília, número 7, 2011, pp. 5-38.

recorte general previsto en la fórmula, que en documentos anteriores.

A los efectos de asegurar que la designación de productos sensibles no comprometiera los niveles - objetivo de mejora del acceso a mercados, en este documento como también lo hacían los anteriores, se preveía el establecimiento de una forma de compensación. Esta forma de compensación era a través o de la creación de contingentes arancelarios con aranceles intra - cuota más reducidos y aranceles extra-cuota equivalentes a los tipos normales resultantes de la reducción arancelaria, o del incremento de los cupos arancelarios ya existentes, cuya magnitud fuese inversamente proporcional al desvío respecto a la reducción arancelaria general. Además, se establecía como variable de referencia para calcular el incremento en las cuotas arancelarias de los países desarrollados, el nivel de consumo doméstico, reafirmando la propuesta del Paquete de Julio de 2008 al respecto ${ }^{7}$. Para los países en desarrollo, la ampliación de los contingentes se coloca en dos tercios del volumen de los países desarrollados. De este modo, se simplificaba el proceso, al no recurrir a complejas fórmulas matemáticas, como lo venía proponiendo la Unión Europea.

Otra de las posibilidades de flexibilización de los recortes arancelarios era la designación de productos especiales por parte de los países en desarrollo. El texto de diciembre de 2008 mantenía la estructura de

7 Esa nueva "oportunidad de acceso" sería del 4 por ciento del consumo interno si se aplica plenamente el apartamiento de dos tercios, del 3,5 por ciento si sólo se efectúa la mitad de la reducción, o del 3 por ciento si se aplica el apartamiento menor, de un tercio. 
Artigo original

Hegemonia - Revista Eletrônica de Relações Internacionais do Centro Universitário Unieuro

ISSN: 1809-1261

UNIEURO, Brasília, número 7, 2011, pp. 5-38.

dos estratos, pero suprimía la opción de que ningún producto quedara exento de reducciones arancelarias. Además, establecía que los países podían designar hasta el $12 \%$ de los productos como productos especiales, de acuerdo a indicadores basados en los criterios de seguridad alimentaria, seguridad de los medios de subsistencia, y desarrollo rural. Asimismo, contemplaba la posibilidad de que hasta el $5 \%$ de estos productos especiales quedase exento de la obligación de hacer reducciones arancelarias. Pero en todo caso, los recortes arancelarios tenían que ser en promedio del $11 \%$. Señalaba, asimismo, la posibilidad de un tratamiento especial y diferenciado para los países de reciente adhesión al sistema, en virtud del cual, estos países podían designar como productos especiales hasta un $13 \%$ de su universo arancelario agrícola y el porcentaje promedio de reducción arancelario quedaba en el entorno del $10 \%$.

Tal como se venía negociando, en el marco de las negociaciones de Doha, tanto el algodón como los productos tropicales recibían un tratamiento especial en materia de acceso a mercados. De este modo, para los productos tropicales, se buscaba acelerar la liberalización comercial pero preservando las situaciones de comercio preferencial creadas por acuerdos y convenios bilaterales de larga data. Se sugería para ello, el otorgamiento de franquicias arancelarias - aranceles cero -, si los niveles consolidados eran inferiores a 20\% o reducciones arancelarias de $80 \%$ si estos aranceles consolidados eran superiores. Para aquellos productos tropicales que gozasen de preferencias de larga data se preveía una liberalización más lenta: consistente en un período de aplicación de 10 años, durante los 
Artigo original

Hegemonia - Revista Eletrônica de Relações Internacionais do Centro Universitário Unieuro

ISSN: 1809-1261

UNIEURO, Brasília, número 7, 2011, pp. 5-38.

cuales se establecían reducciones anuales, siendo que los dos primeros años estaban exentos de reducción y en una reducción escalonada con porcentajes de recorte que oscilaban entre el $50 \%$ para los niveles arancelarios consolidados inferiores al $20 \%$ y el $70 \%$ para los niveles superiores al $75 \%$. Se preveía que este tratamiento se otorgara al $65 \%$ de los productos tropicales incluidos en la lista negociada, que se encontraba en el Anexo $\mathrm{H}$ del documento.

El proyecto también se refiere a las salvaguardias. Por un lado, trata el mecanismo de la Clausula de Salvaguardias Especiales para la Agricultura del artículo 9 del Acuerdo de Agricultura. En este sentido, lo propuesto poco se apartaba de las revisiones anteriores: un plazo para la eliminación total del mecanismo de 7 años en el caso de los países desarrollados, la reducción inmediata del número de productos que se podía acoger al mismo, al $1 \%$ para el caso de los países desarrollados, al 2,5\% para los países en desarrollo (manteniéndose incluso este nivel luego del período de 7 años) y $5 \%$ para las pequeñas economías vulnerables pero en un período de 12 años, la no aplicación de aranceles superiores a los consolidados durante la aplicación del mecanismo. Además se reserva este mecanismo para aquellos productos que estuviesen sujetos a aranceles variables, a regímenes de licencias de importación discrecionales, contingentes o prohibiciones de las importaciones convertidos en aranceles en la Ronda Uruguay.

Por otro lado, trata lo que sería la nueva creación de la Ronda de Doha: la adopción de un nuevo Mecanismo de Salvaguardia Especial. Según lo establecido en este documento y en los anteriores, este 
Artigo original

Hegemonia - Revista Eletrônica de Relações Internacionais do Centro Universitário Unieuro

ISSN: 1809-1261

UNIEURO, Brasília, número 7, 2011, pp. 5-38.

mecanismo beneficiaría a los países en desarrollo, quienes quedaban habilitados a proteger temporalmente a sus productores nacionales mediante este mecanismo. En todo caso, este mecanismo podía ser aplicado a todos los productos agrícolas. Además, se proponía que la habilitación de este mecanismo quedaba sujeta a dos mecanismos gatillo: uno por volumen y otro por precio. El primer mecanismo de gatillo suponía que si se producía un incremento desmesurado del volumen de las importaciones respecto al existente en el trienio anterior a la entrada de vigor de la reforma, los países quedaban habilitados a imponer derechos adicionales sobre los aranceles aplicados, que podían ser de hasta el $50 \%$ en caso de que el incremento en el volumen de las importaciones fuese superior al $35 \%$. En cuanto al segundo, se establecía que si se producía una caída del $85 \%$ en el precio $\mathrm{ClF}$ del producto importado respecto a un precio de referencia fijado para el producto para la nación más favorecida, los países podían imponer un derecho adicional equivalente al $85 \%$ de la diferencia entre el precio $\mathrm{CIF}$ al que se hace la importación y el precio de referencia. Además, se proponía que mientras en el gatillo - volumen, la habilitación de este mecanismo debía tener una duración anual (12 meses) y no podía ser aplicado en más de dos períodos consecutivos; en el caso del gatillo - precio, la aplicación debía hacerse envío por envío.

En este pilar, se proponía también un tratamiento más que diferenciado para los países considerados menos avanzados. $Y$ en esto se reiteraba lo que ya estaba presente en las versiones y revisiones anteriores. Pues estos países quedaban exentos de tener que reducir sus aranceles. Además, se contemplaba que estos países 
Artigo original

Hegemonia - Revista Eletrônica de Relações Internacionais do Centro Universitário Unieuro

ISSN: 1809-1261

UNIEURO, Brasília, número 7, 2011, pp. 5-38.

se beneficiaran de un acceso a los mercados libre de aranceles y contingentes para el $97 \%$ de sus productos originarios y que para esos productos se aplicaran normas de origen preferenciales sencillas Por último, se contemplaba que la supervisión de este tratamiento y de los progresos de estos países quedaba a cargo del Comité de Comercio y Desarrollo.

\section{El segundo pilar: las Ayudas o Subvenciones internas}

Como en las versiones anteriores, en el texto de diciembre de 2008, y siguiendo con los mandatos establecidos en la Declaración Ministerial de Doha y en Esquema de las Modalidades de agosto de 2004, se establecía el compromiso de promover reducciones sustanciales de las ayudas internas, causantes de distorsión de los mercados, en la medida que implicaban descenso de los precios mundiales de los productos agrícolas. En este sentido, no se buscaba la eliminación total de estos programas. Por lo que contemplaba el mantenimiento de una cuantía pequeña o mínima de ayuda que no podía ser superior al 2,5\% del valor de la producción agrícola para los países desarrollados y al 6,7\% de dicho valor en el caso de los países en desarrollo. Sin embargo, permitía de un modo ilimitado, las ayudas y subvenciones internas que carecían de efecto distorsionante ni sobre el comercio, ni sobre la producción. Dentro de esta amplia gama de programas de ayudas permitidos abiertamente, se encontraban las ayudas al desarrollo, la infraestructura, la investigación, la extensión agraria, el reajuste estructural, etc. 
Artigo original

Hegemonia - Revista Eletrônica de Relações Internacionais do Centro Universitário Unieuro

ISSN: 1809-1261

UNIEURO, Brasília, número 7, 2011, pp. 5-38.

Esta nueva versión del proyecto sobre las Modalidades, contemplaba una nueva definición de la Ayuda Global causante de distorsión del comercio. Bajo esta denominación no sólo quedaban incluidas los programas incluidos dentro del compartimiento "ambar" ${ }^{8}$, medidos a traves de la MGA, sino además los niveles de mínimis de ayuda permitida y aquellas ayudas del compartimiento azul ${ }^{9}$.

Para esta ayuda se establecía el compromiso de tener que realizar las reducciones más profundas de las cifras de ayuda otorgada en el príodo 1995-2000. Como en los documentos anteriores, se establecía que estas reducciones operaran simultáneamente mediante tres niveles de limitaciones. El primero de los cuales suponía que cada subcategoría que integra esta mega-categoría quedara también reducida o limitada. En segundo lugar, en cada una de las subcategoría se preveía una limitación de la ayuda por producto específicamente. Y por último, se preveía el establecimiento de reducciónes de las cantidades permitidas con respecto a las tres subcategorías juntas.

Asimismo, se estimaba que los recortes se realizaran por dos métodos que implicaban reajuestes en los límites máximos permitidos que podían o no incidir en el gasto real. El primero de ellos implicaba clasificar en tres estratos los niveles de ayuda global distorsionante del comercio: un estrato superior con un monto umbral de $60 \mathrm{mil}$

8 Aquellos que causaban mayor distorsión por sus efectos directos tanto sobre los precios como sobre la producción

9 Estas eran las causantes de la menor distorsión en virtud de las condicionantes que se le imponían 
Artigo original

Hegemonia - Revista Eletrônica de Relações Internacionais do Centro Universitário Unieuro

ISSN: 1809-1261

UNIEURO, Brasília, número 7, 2011, pp. 5-38.

millones de dólares, un estrato intermedio, con un monto umbral ubicado entre los $10 \mathrm{mil}$ y los $60 \mathrm{mil}$ millones de dolares y uno inferior, con una cifra de ayuda inferior a los 10 mil millones de dólares. Tal como se había previsto para las reducciones arancelarias en el primer pilar, las ayudas del estrato superior debían sufrir las disminuciones más profundas. El segundo de los métodos, suponía el establecimiento de límites máximos tanto para las ayudas de mínimis y para las del compartimiento azul como para las ayudas por producto específico.

De este modo, a grandes rasgos, para esta categoría de ayudas, sin estar indicado expresamente en el texto, se preveía que entre los países desarrollados, la Unión Europea ${ }^{10}$ y J apón, eran los que debían realizar los mayores porcentajes de recorte: $80 \%$ en el caso de la Unión Europea y $75 \%$ en el caso japonés ${ }^{11}$. Para los EEUU ${ }^{12}$ y para el resto de los países desarrollados, cuyas ayudas ascendían a menos de 10 mil millones de dolares, este porcentaje de reducción era de $70 \%$ y $55 \%$ respectivamente. Además, se preveía una reducción inmediata el 33\% como "aportación inicial" de los montos de las ayudas para los EEUU, la Unión Europea y Japón y del 25\% para el resto de los países desarrollados.

10 Por haber otorgado ayudas en el período de base superiores a los 60 mil millones de dólares.

11 Si bien el monto de la ayuda global distorsionante del comercio japonés era similar al otorgado por el gobierno de los EEUU (situándose entre los 10 y los 60 mil millones de dólares y por lo tanto el porcentaje de recorte era equivalente del de los EEUU, en el caso japonés existía un $5 \%$ adicional de reducción por el peso que tenía esta ayuda global en el valor de la producción agrícola japonesa, que ascendía a el $40 \%$.

12 Entre 1995-2000, el monto de la ayuda de este país ascendió a los 48,2 mil millones de dólares. 
Artigo original

Hegemonia - Revista Eletrônica de Relações Internacionais do Centro Universitário Unieuro

ISSN: 1809-1261

UNIEURO, Brasília, número 7, 2011, pp. 5-38.

En cambio, para los países en desarrollo se contemplaba una situación diferente. Pues para aquellos países en desarrollo como los países menos desarrollados y los importadores netos de alimentos, que no tuviesen compromisos de reducción en materia de MGA, tampoco tenían que reducir sus montos totales de Ayuda Global distorsionante del comercio, pero sí debían mantenerse dentro de sus niveles de base. En cambio, para aquellos países ne desarrollo que si los tuvieran, el compromiso de reducción ascendía al 36,3\%.

Similar tratamiento al otorgado a los países en desarrollo, se señalaba para los nuevos miembros del sistema y para aquellos con muy bajos ingresos.

Además se preveía que estas reducciones se debían realizar en un período de 5 años para los países desarrollados y de 8 años para los países en desarrollo.

En materia de las ayudas del denominado compartimiento ámbar, siguiendo con lo ya señalado, el texto preveía una reducción de la MGA total final consolidada, una limitación de la MGA por producto específico y una nueva base de cálculo de esta ayuda, teniéndo en cuenta la inflación y en el caso de los países en desarrollo, los incrementos abruptos en el precio de los alimentos.

Con relación a la MGA Total final consolidada, el texto bajo análisis no presentaba grandes variaciones respecto a las versiones anteriores. Como en el caso de la mega - categoría de Ayuda total distorsionante 
Artigo original

Hegemonia - Revista Eletrônica de Relações Internacionais do Centro Universitário Unieuro

ISSN: 1809-1261

UNIEURO, Brasília, número 7, 2011, pp. 5-38.

del comercio, se establecía una clasificación de los montos de la MGA total final consolidada en tres bandas: una banda superior con una cifra - umbral de 40 mil millones de dólares, una banda intermedia situada entre los 15 mil millones y los 40 mil millones de dólares y una banda inferior, con cifras menores a los 15 mil millones de dólares. Además, preveía porcentajes de reducción más elevados para la franja superior de la MGA Total final consolidada. De este modo, sin tampoco quedar precisado en los textos, se preveía que la Unión Europea y Japón debían realizar los mayores recortes - 70\% por situarse ambos en el estrato superior, los EEUU redujeran sus montos de MGA total final consolidada en un $60 \%$ por estar en la banda intermedia mientras que los demás países desarrollados, sólo lo debían hacer en un $45 \%$, excepto cuando el peso de esta ayuda fuese superior al $40 \%$ del valor de su producción agrícola debiendo entonces realizar un recorte mayor - un 7,5\% adicional al correspondiente a su franja - ${ }^{13}$. Además, se preveía que tanto la Unión Europea como los EEUU y Japón debían realizar una aportación inicial equivalente al $25 \%$ de la reducción correspondiente. Se contemplaba que el plazo para realizar estos recortes era el mismo que para las reducciones de la Ayuda Total distorsionante del comercio.

Para los países en desarrollo, el tratamiento especial y diferenciado en materia a MGA Total Final consolidada preveía que aquellos países que ya tenían compromisos de reducción de la MGA en virtud del Acuerdo de Agricultura, debían realizar reducciones de su MGA total

13 En esta situación, estan países como Noruega o Suiza, integrantes del G-10. 
Artigo original

Hegemonia - Revista Eletrônica de Relações Internacionais do Centro Universitário Unieuro

ISSN: 1809-1261

UNIEURO, Brasília, número 7, 2011, pp. 5-38.

final consolidada en un $30 \%$, en un período de 8 años. Pero además, se preveía que para los países en desarrollo con montos de MGA inferiores a los 100 millones de dólares, o para aquellos que fuesen importadores netos de productos alimenticios, todos ellos quedaban exentos del compromiso de tener que realizar recortes en sus niveles de MGA total final consolidada.

Asimismo, marcaba un trato diferencial para los miembros de reciente incorporación al sistema, especialmente para los de muy reciente adhesión y los de bajos ingresos, que preveía también que quedaran exentos del compromiso de reducción de la MGA total final consolidada. Además a algunos de ellos, se les permitía excluir las subvenciones a la inversión del cálculo de la MGA.

En relación a los limites máximos a la MGA por productos específicos se establecía que la misma no podía ser superior a la que realmente había sido otorgada en promedio por los países desarrollados, en el período 1995-2000. Pero en el caso de los países desarrollados preveía un tratamiento diferente para los EEUU. Pues en este caso, permitía que el cálculo de la MGA por producto específico se realizara teniendo en cuenta la aplicación proporcional del promedio de la MGA para cada producto otorgada en cada año del período 1995-2004 con respecto al promedio de la MGA total por producto específico para el período 1995-2000.

Por otra parte, para los países en desarrollo, establecía tres opciones:

i. El límite máximo era el promedio de la MGA por producto específico durante el período 1995-2000 (o 1995-2004) 
Artigo original

Hegemonia - Revista Eletrônica de Relações Internacionais do Centro Universitário Unieuro

ISSN: 1809-1261

UNIEURO, Brasília, número 7, 2011, pp. 5-38.

ii. El doble del nivel de mínimis por producto específico en dicho período.

iii. El $20 \%$ de la MGA total final consolidada durante el período considerado como base.

En cuanto al nivel de mínimis de las ayudas del compartimiento ambar permitida, el texto en cuestión tampoco implicaba grandes variaciones con relación a las versiones anteriores. Para los países desarrollados proponía una reducción del 50\% desde el primer día. De este modo, el nivel de mínimis permitido de ayuda pasaba a ser el $2,5 \%$ del valor de la producción. Y para los países en desarrollo, con compromisos de reducir su MGA, se preveía un recorte del 33\% hasta llegar al 6,7\% del valor de la producción. Para aquellos países en desarrollo, sin compromisos de reducción o con toda la ayuda destinada a "agricultores de subsistencia y pobres de recurosos o países importadores netos de alimentos, quedaban exentos de compromisos de reducción de sus nieles de mínimis. Exenciones similares se aplicaban para los países de muy reciente incorporación al sistema y para algunos de los países con bajos ingresos. Para aquellos países de reciente adhesión con niveles de mínimis del 5\% del valor de la producciónsólo debían recortar estas ayudas en un $15 \%$.

El otro tipo de ayudas distorsionantes del comercio son aquellas que entraban dentro del compartimiento azul. Con relación a éstas, la revisión de diciembre de 2008 planteaba una nueva definición de dicho compartimiento, en el entendido que el vigente causaba una limitada distorsión del comercio, en la medida que suponía la 
Artigo original

Hegemonia - Revista Eletrônica de Relações Internacionais do Centro Universitário Unieuro

ISSN: 1809-1261

UNIEURO, Brasília, número 7, 2011, pp. 5-38.

realización de pagos directos a los agricultores sobre la base de la cantidad de animales que tienen o de la superficie cultivada, pero que implicaban límites a la producción, con el objetivo de evitar la superabundancia de producción. Por lo que en la nueva definición del contenido de compartimiento azul, sólo se debía incluir aquellos pagos que no requerían producción sino que se basasen en una cantidad fija de producción anterior. Además proponía que cada país decidiera qué tipo de ayuda del compartimiento azul emplearía, para todos los productos. Las posibles excepciones debían ser aprobadas cuando se acordasen las listas de compromisos. Pero en todo caso, un producto sólo podría recibir un tipo de ayuda de este compartimiento.

Se mantenía que las ayudas de este compartimiento debían tener un límite máximo que en el caso de los países desarrollados era del 2,5\% del valor de la producción durante el período base (1995 2000), excepto si la ayuda de dicho compartimiento era superior al $40 \%$ de la ayuda causante de distorsión al comercio, en cuyo caso, se reduciría en el mismo porcentaje que la MGA en el plazo de dos años. Para los países en desarrollo y los países de reciente incorporación al sistema, el límite máximo que podían alcanzar las ayudas del compartimiento azul era del $5 \%$ del valor de la producción del período base, para el cual se planteaba una cierta flexibilidad. Esta flexibilidad relativa se debía a que para aquellos países en desarrollo que hubiesen trasladado al compartimiento azul, ayudas que anteseran consideradas al calcular la MGA luego de finalizada la ronda de negociación, el período de base debía ser el período más reciente. 
Artigo original

Hegemonia - Revista Eletrônica de Relações Internacionais do Centro Universitário Unieuro

ISSN: 1809-1261

UNIEURO, Brasília, número 7, 2011, pp. 5-38.

Asimismo, se planteaban cómo se debían establecer límites a las ayudas del compartimiento azul por productos específicos otorgadas por parte de los países desarrollados, señalando que los límites se fijarían teniendo en cuenta el valor del gasto medio en el período base (1995-2000), indicando que en caso de no haberse otorgado ayuda durante ese período, se debían tomar el valor promedio del gasto realizado en los años notificados dentro de ese período, a condición de que hubiesen tres años consecutivos notificados.

Para el caso de los EEUU, los límites debían ser superiores en un 10 o $20 \%$ a las estimaciones de gastos máximos permitidos por la Farm Bill de 2002.

Por último, en lo referente a la ayuda no distorsionante del comercio, que entraba dentro del compartimiento verde, el texto planteaba revisiones, en particular en lo que referente a la verdadera desconexión de este tipo de ayuda respecto a los niveles de producción, la constitución de existencias de productos alimenticios por parte de los países en desarrollo y a la vigilancia y supervisión más estrecha. Ello preveía una modificación de las disposiciones del Acuerdo sobre la Agricultura en su anexo 2, para permitir la aplicación de programas de desarrollo por parte de los países en desarrollo y para reforzar la rigurosidad de los criterios en los países desarrollados. En este sentido, si bien en la revisión de julio de 2008, se perfeccionaban las disposiciones respecto a períodos de base fijos e invariables para los programas de ayuda a los ingresos, reajuste estructural y asistencia regional, en el texto de diciembre de 2008, se perfeccionaban las condiciones para que algunos tipos de intervención 
Artigo original

Hegemonia - Revista Eletrônica de Relações Internacionais do Centro Universitário Unieuro

ISSN: 1809-1261

UNIEURO, Brasília, número 7, 2011, pp. 5-38.

gubernamental en los países en desarrollo se contabilizasen entre estas ayudas no distorsionantes. Estas condiciones se referían a las compras para constitución de existencias para luchar contra el hambre y la pobreza, que suponían compras que se realizaban a precios superiores a los del mercado.

En el siguiente cuadro se muestran los diferentes cambios que de acuerdo al texto analizado se debían incluir en el Acuerdo sobre la Agricultura.

Anexo 2 del Acuerdo sobre Modificación propuesta Agricultura

Servicios generales

Incluir políticas y servicios relacionados con el asentamiento de agricultores, reforma agraria, desarrollo rural y seguridad de los medios de subsistencia rural en los países en desarrollo.

Constitución de existencia Clarificar que no se exigiría públicas con fines de seguridad contabilizar en la MGA la alimentaria adquisición de existencias de productos alimenticios por parte de los países en desarrollo con objeto de ayudar a los productores con ingresos bajos o pobres.

Clarificar que la adquisición de productos alimenticios a precios 
Artigo original

Hegemonia - Revista Eletrônica de Relações Internacionais do Centro Universitário Unieuro

ISSN: 1809-1261

UNIEURO, Brasília, número 7, 2011, pp. 5-38.

subvencionados cuando se

obtengan en general de

productores con ingresos bajos o

pobres de los Países en desarrollo

con objeto de luchar contra el

hambre y la pobreza.

Ayuda a los ingresos Clarificar que el período de base desconectada definido debe ser histórico $y$, además de fijo e invariable.

Asistencia para el reajuste Autorizar transferencia de estructural otorgadas mediante derechos a la ayuda existente ayudas a la inversión entre productores o propietarios de la tierra.

Permitir actualización excepcional del período de base, a condición de que las expectativas de los productores y las decisiones sobre la producción no se vean afectadas.

Pagos en el marco de programas Permitir que los miembros que no de asistencia regional hayan utilizado anteriormente este tipo de pagos, puedan establecer un período de base apropiado que siempre que no se basen en una utilización de factores o producción futuras, no sea necesario el basarse en una determinada trayectoria histórica 
Artigo original

Hegemonia - Revista Eletrônica de Relações Internacionais do Centro Universitário Unieuro

ISSN: 1809-1261

UNIEURO, Brasília, número 7, 2011, pp. 5-38.

existente, pero que sean fijo e invariable así como notificado.

Pagos (efectuados directamente Indicar que los PeD pueden - a través de la participación efectuar pagos a los productores financiera del gobierno en planes aun cuando la pérdida de inferior de seguros de las cosechas) en al $30 \%$ de la producción media concepto de socorros en caso de de los 5 años precedentes o de desastres naturales. un promedio olímpico Indicar que el derecho a percibir esos pagos se determinará por una pérdida de producción superior al 30\% del promedio de la producción en un período que se demuestre actuarialmente apropiado

Clarificar que los pagos se aplican también para cubrir pérdidas de cosechas o la destrucción de animales o cosechas.

Pagos en el marco de programas Eximir a los Países en Desarrollo de asistencia regional de la condición de que las regiones desfavorecidas sean una zona geográfica continua.

Clarificar que la producción agrícola a que se refiere, comprende la producción ganadera. 
Artigo original

Hegemonia - Revista Eletrônica de Relações Internacionais do Centro Universitário Unieuro

ISSN: 1809-1261

UNIEURO, Brasília, número 7, 2011, pp. 5-38.

Como en el caso del primer pilar, el algodón recibía un tratamiento especial también este pilar. De este modo, la última revisión del proyecto sobre modalidades de julio de 2006 preveía que la ayuda interna causante de distorsión del comercio, en este producto, debía reducirse en una proporción mayor a la que se beneficiaban los otros productos del sector. Para ello, se hacía referencia a la fórmula propuesta en 2006, por los Cuatro del Algodón. Del mismo modo, establecía que la ayuda del compartimiento azul quedaba limitada a un tercio de lo que sería el límite normal. Además, se contemplaba un tratamiento diferenciado para los países en desarrollo que tuviesen compromisos de reducción de los niveles de ayuda en materia de MGA y del compartimiento azul, que suponía porcentajes de reducción inferiores y plazos mayores.

\section{EI tercer pilar: la política de competencia de las exportaciones.}

Siguiendo con lo establecido en la Conferencia Ministerial de Hong Kong de 2005, las Modalidades debían asegurar la eliminación total de toda forma de subvención a las exportaciones. Ello suponía no sólo elminar los subsidios a la exportación, asegurando que los países en desarrollo continuasen accediendo de un modo continuo al compromiso del artículo 9 inciso 4 del Acuerdo sobre la Agricultura ${ }^{14}$.

14 "Durante el período de aplicación, los países en desarrollo Miembros no estarán obligados a contraer compromisos respecto de las subvenciones a la exportación enumeradas en los apartados d) - subvenciones para reducir los costos de comercialización de las exportaciones de productos agropecuarios (excepto los servicios de asesoramiento y promoción de exportaciones de amplia disponibilidad) incluidos los costos de manipulación, perfeccionamiento y otros gastos de transformación y los costos de los transportes y fletes internacionales- y e) - las tarifas de los transportes internos de los envíos de exportación establecidos o impuestos por los gobiernos en condiciones más favaroables que los envíos internos - del párrafo 1 supra, siempre que dichas subvenciones no se apliquen de manera que se eludan los compromisos de reducción" (Organización Mundial del Comercio, 1994). 
Artigo original

Hegemonia - Revista Eletrônica de Relações Internacionais do Centro Universitário Unieuro

ISSN: 1809-1261

UNIEURO, Brasília, número 7, 2011, pp. 5-38.

Implicaba también mejorar las disciplinas para impedir la evasión de las normas en materia de ayuda alimentaria internacional, créditos y garantías a la exportación y la acción exportadora de las empresas comercializadoras del Estado.

De este modo, la revisión del poyecto de Modalidades de diciembre de 2008 consagraba la eliminación de las subvenciones a la exportación para el año 2013, con una reducción del 50\% a fines de 2010, de las cantidades otorgadas como subvenciones en el período 2003-2005, en el caso de los países desarrollados. Para los países en desarrollo, esta eliminación total debía producirse hacia el año 2016, mediante un proceso de reducción en tramos iguales anuales. Además, se establecía que el compromiso de acceso continuo a la cláusula 4 del artículo 9, quedaba asegurado hasta el año 2021. El texto aseguraba el compromiso de exención del compromiso de eliminación de las subvenciones a la exportación para los países importadores netos de alimentos y los países menos avanzados.

En materia de créditos a la exportación, garantías de créditos a la exportación o programas de seguro, el documento establecía que los mismos quedaban sometidos a disciplinas para evitar las subvenciones encubiertas. De este modo, estas disciplinas suponían someter a los programas de financiamiento de las exportaciones a condiciones que se referían a limitar el período de reembolso a 180 días y a garantizar que los programas se autofinancien en un plazo de 4 años en el caso de los países desarrollados y de 6 años en el de los países en desarrollo. 
Artigo original

Hegemonia - Revista Eletrônica de Relações Internacionais do Centro Universitário Unieuro

ISSN: 1809-1261

UNIEURO, Brasília, número 7, 2011, pp. 5-38.

Para los países en desarrollo que concediesen créditos, el plazo máximo de 180 días para el reembolso debía aplicarse en tres etapas durante un periodo de 4 años. Por otra parte, en el caso de los países menos avanzadis y aquellos países en desarrollo importadores netos de alimentos, el plazo de reembolso se ampliaba a entre 360 y 540 días

Por su parte, en cuanto a las empresas comerciales del Estado que exportasen productos agropecuarios, se contemplaba que sus acciones estuviesen sometidas a reglas a fin de eliminar los poderes monopólicos de estas empresas, salvo que cuatro excepciones:

i. dicha empresa fuese notificada a la OMC y no eludiera las disciplinas

ii. dicha empresa perteneciese a un país en desarrollo ejerciendo un poder monopólico para preservar la estabilidad de los precios al consumo interno y/o para garantizar la seguridad alimentaria,

iii. dicha empresa perteneciese a un países menos avanzado y/o a una economía pequeña vulnerable

iv. las exportaciones de dicha empresa fuesen por tres años consecuentivos inferiores al $5 \%$.

En lo referente a la ayuda alimentaria internacional, señalaba que la ayuda alimentaria de emergencia debía considerada dentro de un compartimiento considerado seguro, con disciplinas más flexibles. Las declaraciones de situación de emergencias debían ser realizadas por las organizaciones internacionales competentes -las Naciones Unidas, 
Artigo original

Hegemonia - Revista Eletrônica de Relações Internacionais do Centro Universitário Unieuro

ISSN: 1809-1261

UNIEURO, Brasília, número 7, 2011, pp. 5-38.

el Programa Mundial de Alimentos, la Cruz Roja-. Además, la evaluación de las necesidades debía ser coordinada bajo los auspicios de los organismos internacionales competentes. Asimismo, en este caso, no quedaba sujeta a monetización de la ayuda salvo que la ayuda fuese otorgada a un país menos avanzado cuando fuese necesario para efectos de transporte y entrega. Para aquella ayuda no urgente se preveía que quedara sometida a reglas a los efectos de evitar que desviara comercio. Por lo que se proponía que quedara sujera a una evaluación de las necesidades por parte de un organismo internacional del sistema de Naciones Unidas. Asimismo se contemplaba dar prioridad al gobierno receptor con relación a todas las operaciones de ayuda alimentaria, haciendo hincapie en la evaluación de las necesidades. Al mismo tiempo se señalaba que cuando esta evaluación fuese realizada por ONGs la decisión última de conceder o no la ayuda alimentaria quedaba a cargo de la ONU. La monetización o la venta de los productos donados para conseguir fondos destinados a ayuda quedaba sometida a regulaciones.

En lo referente a las prohibiciones y/o restricciones a la exportación se pretendía reforzar las reglas con relación al establecimiento de nuevas restriciones a la exportación, en condiciones de mayor transparencia y vigilancia, previéndose incluso el mecanismo de las consultas.

\section{Reflrexiones finales}

A modo de reflexión final, se puede decir que el objetivo de este artículo simplemente consistía en presentar de un modo analítico las 
Artigo original

Hegemonia - Revista Eletrônica de Relações Internacionais do Centro Universitário Unieuro

ISSN: 1809-1261

UNIEURO, Brasília, número 7, 2011, pp. 5-38.

características principales del contenido de la cuarta revisión del Proyecto Falconer sobre las Modalidades, presentado por el Presidente Falconer en julio de 2007. Asimismo, como todas las revisiones anteriores y el proyecto anterior de Modalidades, esta cuarta revisión del texto no significaba en ningún caso, un acuerdo definitivo sobre el contenido de las Modalidades. Ello queda en evidencia en que las negociaciones continuaron hasta el día de hoy. Los trabajos y tareas de negociación en el marco de las sesiones extraordinarias del Comité de Agricultura han continuado en el plano multilateral, mediante rondas de consultas para buscar el consenso necesario para precisar el contenido de las modalidades en aquellos temas del temario incluido en el texto que se analizó, en los que el consenso estaba pendiente. Estas consultasvse realizaron a los diferentes grupos de Estados que se han constituido, a lo largo del largo proceso de negociación, en las partes activas de la misma, siendo creadores de las propuestas complejas o sensillas, globales o individuales, según fuesen abarcadoras de todos o algunos de los temas de la agenda compleja de negociación, y que representaban las posturas generales de los distintos miembros del Sistema Multilateral de Comercio Asimismo, estas consultas pusieron en evidencia que algunos casos se dependía de decisiones políticas y en otros casos, sólo era cuestión de definiciones técnicas.

Asimismo, el análisis de esta cuarta revisión muestra que los temas del primer pilar son los temas más complejos en alcanzar un consenso, sobre todo aquellos que tienen que ver con las excepciones a los recortes arancelarios previstos, es decir la designación de productos sensibles, el mantenimiento del sistema de cláusula de 
Artigo original

Hegemonia - Revista Eletrônica de Relações Internacionais do Centro Universitário Unieuro

ISSN: 1809-1261

UNIEURO, Brasília, número 7, 2011, pp. 5-38.

salvaguarda así como aquellos aspectos que marcaban un cambio en el carácter del tratamiento especial y diferenciado a favor de los países en desarrollo (países menos avanzados y economías pequeñas y vulnerables), con la creación de un nuevo Mecanismo de Salvaguardia especial y la posibilidad de designación un porcentaje de productos agrícolas como productos especiales.

Pero tambien resultaba dificil la negociación en el segundo pilar, aquel que suponía reducciones sustanciales en los montos de las ayudas internas, en especial en aquellos temas, las reducciones de las ayudas internas distorsionantes del comercio por producto específico, los montos de ayuda distorsionante "de mínimis" por producto específico, los programas de ayuda interna que entran en el denominado compartimiento azul y la definición de los requisitos que asegurasen que las ayudas internas que no estuvisen sujetos al compromiso de reducción fuesen libre de causar efectos distorsionantes en el comercio.

Si bien el consenso ya estaba alcanzado en lo referente a la eliminación total de las subvenciones a la exportación en el sector para el año 2013 para los países desarrollados y 2016 para los países en desarrollo, los temas del tercer pilar que resultaban de dificil consenso eran aquellos que tenían que ver con las reglamentaciones de los sistemas de créditos, garantías de créditos y seguros a las exportaciones y con la situación monopólica de las empresas comercializadoras del Estado, entre otros. 
Artigo original

Hegemonia - Revista Eletrônica de Relações Internacionais do Centro Universitário Unieuro

ISSN: 1809-1261

UNIEURO, Brasília, número 7, 2011, pp. 5-38.

\section{BI BLI OGRAFÍ A}

Libros

Baltzer, $\mathrm{K}$ et al (2009). Trade Liberalization in the Doha Round. West Laffayette. GTAP

Berlinski, J. y Stancanelli, N. (2010). Los acuerdos comerciales. Reflexiones desde un enfoque argentina. CARI-CEI. Buenos Aires.

Revistas

Bhala, Raj (2010). "Resurrecting the Doha Round: Devilish, Details, Grand Themes, and China too", en Texas International Law Journal, Vol 45.

Hertel, Th et al (2010). "Potential Implications of a Special Safeguards Mechanism in the World Trade Organization" en The World Bank Economic Review. Vol 24 (2).

Working Papers

Adler, M et al (2009). What's on the table. The Doha Round as of August 2009. PIEE. Working Papers Washington. Agosto 2009. 
Artigo original

Hegemonia - Revista Eletrônica de Relações Internacionais do Centro Universitário Unieuro

ISSN: 1809-1261

UNIEURO, Brasília, número 7, 2011, pp. 5-38.

Gouel Christophe, et al (2010) "The art of exceptions: sensitive products in the Doha Negotiations". CEPII Working Papers 2010-20. Paris, Octubre 2010.

Hufbauer, Gary Clyde; Lawrence, Robert (2010). Let's Make a deal: How to Bring Doha Talks to a close". PIIE Working Papers. Washington, Agosto 2010.

Jean, S. et al (2010). Formulas and flexibility in trade negotiations: sensitive agricultural products in the WTO's Doha agenda. World Bank Policy Research Working Papers 5200. World Bank. Washington, D.C.

Documentos

OMC (Organización Mundial del Comercio). 2008a. Revised Draft Modalities for Agriculture. Committee on Agriculture, Special Session, Jul. (TN/AG/W/4/Rev.4).

. 2008b. Revised Draft Modalities for Agriculture. Committee on Agriculture, Special Session, Dic. (TN/AG/W/4/Rev.4).

2008c. Guia No Oficial del Proyecto Revisado de Modalidades Agricultura. Dic. 